\title{
Die Macht weißer Stimmzettel
}

\section{Ein Gedankenspiel zur Bundestagswahl}

\author{
Jan-Philipp Küppers, E. W. Udo Küppers
}

\begin{abstract}
KERNAUSSAGE
Die Politiker haben die nachhaltige gesellschaftliche Weiterentwicklung nicht mehr im Griff! Schaden von den Bürgern abzuwenden, wie es die auftragsgemäße politische Pflicht ist, kehrt sich zunehmend ins Gegenteil. Der Umgang mit Krisen befördert Schuldenanhäufung und Arbeitslosigkeit. Wie können Bürger sich gegen unfähige Politiker zur Wehr setzen? Eine „Weisswahl“, nach José Saramagos Parabel über aufbegehrende Bürger einer Stadt, wäre ein probates Mittel, begleitet von kontrollierten Bürger-Regulativen, einer aufrechten öffentlichen Tätigkeit am Gemeinwesen und der Anleitung für den richtigen Umgang mit realen komplexen Problemen.
\end{abstract}

\section{Einleitung}

Die politische Wahl ist der ureigene Ausdruck jedes wahlberechtigten Bürgers, seine Meinung zu den gesellschaftlichen Themen zu bekunden. Und der Bürger sollte sein Recht wahrnehmen! Eine völlig neue gesellschaftliche Dimension hätte der Wahlvorgang allerdings, wenn sich die Wähler einmal weitgehend einig wären und ihren politischen Vertretern die „weißen Stimmzettel“ zeigen. ${ }^{1}$ „Bei einer politischen Wahl erhalten die Politiker eines namenlosen westlichen Landes eine schallende Ohrfeige: Fast alle abgegebenen Stimmzettel sind weiß“ (Saramago, 2009, Buchrückseite).

Auch im realen Leben wäre eine solche Situation ein echter Härtetest jeder Demokratie. Wie gehen Institutionen und deren Repräsentanten mit dem unvorhergesehenen volonté générale um? Der unerwartete Akt der etwas anderen Stimmenabgabe steht für ein Anzeichen von demokratischem Ordnungsverlust, der für gewöhnlich die geballte Staatsmacht herausfordert. Wie würde sich ein derartiges Wahlergebnis in unserer Demokratie gesellschaftlich auswirken? Vor allem aber: Wie würden Politiker auf diese drastische Brüskierung durch die Bürger, auf die Angst politischer Bedeutungslosigkeit, reagieren?

Ist ein Vergleich zwischen politischer Parabel und realer Wahl in einem namentlich bekannten westlichen Land überhaupt statthaft? Parabeln sind nicht selten die Vorläufer kommender realer Ereignisse, die Leser durch symbolisches Geschehen auffordern, Erzähltes als tatsächlich Vorhandenes zu erkennen! Das weiße Übel aus Saramagos imaginärer Gegenwart ist real existent! Sei es durch verschiedene Anzeichen von Ordnungsverlusten wie Finanz- und Wirtschaftskrisen, Terroranschläge wie „Nine-Eleven“, kalkulierten Risi- ken, wie „Deepwater Horizon“ und „Fukushima“ oder eben weiße Stimmzettel.

„Die größten Triumphe feiert sie [die Demokratie, Anm. d. Verf.] bei schönem Wetter" (Teusch 2008, S. 179) - und bei schlechtem Wetter, das sei hier erwähnt, werden ihre demokratischen Institutionen und gewählten „Leistungsträger“ auf die Probe gestellt. Passive Bürger erklären ihr Verhalten nicht zur Wahl zu gehen möglicherweise mit dem symbolischen Charakter des Wahlaktes bzw. mit der Unzulänglichkeit früherer Wahlausgänge. Damit mögen sie Recht haben. Doch irren sie, wenn sie glauben, mit apathischer Ruhe eine Besserung herbeiführen zu können. Denn Nichtwählen ist keine demokratische Option! Periodische Stimmabgaben - das ist den Autoren wichtig - bleiben etwa bei Wahlen in freier Ausführung kein verkettetes Beiwerk. Sie sind ein entscheidendes Kriterium für repräsentative Demokratien. Nur ist bisher nicht festgelegt, wie hoch die Wahlbeteiligung mindestens ausfallen muss ${ }^{2}$ oder wann eine Wahl für gültig oder ungültig zu erklären ist!

\section{Politik jenseits des Bürgerwillens}

Die zu bewältigenden komplexen und hochkomplexen Herausforderungen der Gegenwart, an die die politischen Akteure teils gelassen herangehen, teils akribisch aber fehlgelenkt feilen (siehe „Energiewende“-Chaos), geben in vielerlei Hinsicht ausreichend Anlass für die Bürger, das Tun und Lassen der Politiker mit den daraus entstehenden Folgen und Folgeproblemen deutlicher denn je zu hinterfragen. Denn ein „ewig“ fortgeschriebenes Politikgesetz in unserer Demokratie scheint zunehmend zu wanken:

Die Bürger übertragen politischen Vertretern durch regelmäßige Wablzyklen die Macht in unserer Gesellschaft. Die Politiker werden beauftragt, Schaden vom Volk abzuwenden.

1 Dieser Essay nimmt das nicht gänzlich unwahrscheinliche Ergebnis einer politischen Wahl zum Vorbild, so wie es José Saramago in seinem Roman „Die Stadt der Sehenden“ beschrieben hat. Auch wenn die gegenwärtige bundespolitische Konstellation vor der Wahl im September 2013 kaum Überraschungen nach Saramagos Vorstellungen zulässt, bleibt doch die Erkenntnis: Die Bürger lassen sich nicht mehr alles gefallen. Sie fordern machtvoll von allen Politikern, ihre Bürgermeinungen für gesellschaftliche Änderungen durch die Politiker nicht nur anzuhören, sondern auch in Entwürfen, Planungen und Praxis zu berücksichtigen; und zwar deutlicher als bislang geschehen.

2 In Deutschland könnten bspw. nur 5\% der wahlberechtigten Bürger und Bürgerinnen zur Wahl gehen, ohne dass daraus für die Parteien irgendwelche Lehren zu ziehen wären, geschweige denn die Wahl annulliert werden würde!

Siehe: www.bundeswahlleiter.de/de/glossar/texte/Gueltigkeit_der_ Wahl.html (Stand: März 2008). 
Jedoch ist zu oft das Gegenteil der Fall, ${ }^{3}$ weil belastende Folgen politischer Aktivitäten letztlich wieder auf die Bürger umgelenkt werden!

Je höher die gesellschaftlich belastenden Folgen sind, desto höher ist die Haftung der Bürger. Es wächst und verfestigt sich ein dominanter Teufelskreis zwischen Bürgern und Politikern. Saramago beschreibt das in seiner unnachahmlichen Weise als das harmonische Binom Autorität-Gehorsam (Saramago, 2009, S. 118). Abbildung 1 skizziert diesen verhängnisvollen Teufelskreis als ein Ausschnitt aus weitaus komplexeren Verknüpfungen zwischen Bürger bzw. Gesellschaft und Politiker.

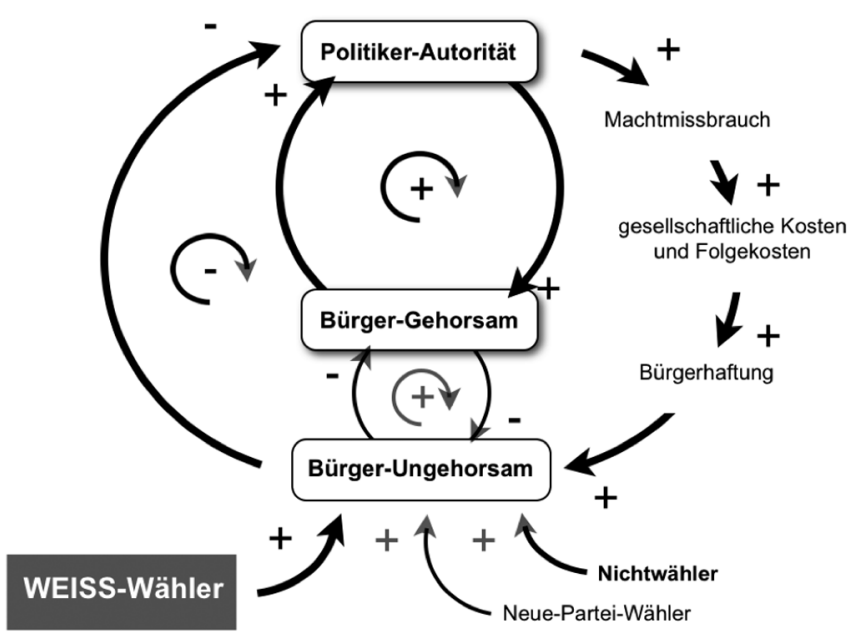

Abbildung 1: Teufelskreis (Wirkungsnetz-Ausschnitt) als „harmonisches Binom “ zwischen Bürger und Politiker mit Regulativen durch Nichtwähler, Neue-Partei-Wähler und WEISS-Wähler.

Die Symbole in diesem Wirkungsnetz haben folgende Bedeutung: $(+)$ und $(-)=$ verstärkte bzw. schwächende Wirkung von... auf. Dreiviertelkreis mit $(+)=$,Teufelskreis“-Funktion. Die Beziehungen schaukeln sich auf oder ab bis zu einem Grenzwert, über den hinaus katastrophale Folgen zu erwarten sind. Dreiviertelkreis mit $(-)=$ Beziehungen führen nachhaltig $\mathrm{zu}$ ausgleichenden Wirkungen mit Angleichung an einem Grenzwert ohne zerstörerische Folgen. Die Wirkungsbeziehungen zwischen Bürgergehorsam und Bürgerungehorsam sind ebenfalls als „Teufelskreis“ zu werten, mit wechselnden Beziehungen, die letztlich auch bis zum Stillstand des einen und Überhöhung des anderen Merkmals führen können. Ohne das jemals ein nachhaltiger, dynamisch stabilisierender Zustand eintreten würde!

Politiker-Autorität an der Macht vertraut auf den Bürgergehorsam. Sofern regierende Politiker die ihnen von den Bürgern übertragene Macht der Handlung „angemessen“, im Sinne allgemein-gesellschaftlicher Fortschritte, nutzen, können sie auf den blinden Bürgergehorsam vertrauen (Kernbeziehung, als Mittelkreis in Abb. 1 skizziert). Sie können es auch dann noch, wenn etwas „aus der Reihe läuft“. Zeigt sich jedoch ein latenter Machtmissbrauch, wie es sich seit geraumer Zeit offenbart, honorieren dies die Bürger auf ihre Weise. „Stuttgart21“ ist hier nur ein herausgestelltes Beispiel des Bürgerunwillens bzw. Ungehorsams (vgl. neben zahlreichen erschienenen Berichten und Kommentaren dazu u.a. Stavrakis, 2013).

Der Wirkungsverlauf im rechten äußeren Halbkreis von Abb. 1 zeigt diese problemverstärkende Wirkung durch eine Politik äußerst geringer Achtsamkeit und Vernetzung, die letztlich den Bürger-Ungehorsam provoziert und aufheizt. Vernetzung bedeutet in diesem Kontext die Fähigkeit der Politiker, reale komplexe Problem-Zusammenhänge ihres Tuns wahrzunehmen und diese mit entsprechenden $-z$. B. kybernetischen - Werkzeugen bzw. Strategien fehlertolerant zu lösen bzw. weiteren Problemen so weit als möglich vorzubeugen.

Bürger-Ungehorsam bahnt sich auch den Weg über die Wahl neuer Parteien wie die „Piraten“ oder die eurokritische „Alternative für Deutschland“. Beide Parteien werden - wenn sie Direktmandate zum Bundestag erzielen bzw. die 5-\%Hürde überspringen - nur marginalen Einfluss auf die Zielausrichtung der „großen“ etablierten Parteien bzw. deren Parteienkoalitionen haben. Davon sind beide Autoren überzeugt. Bürger-Ungehorsam zeigt sich außerdem über Nichtwähler, deren Anteil bei der letzten Bundestagswahl 2009 beachtliche $30 \%$ erreichte (siehe Anmerkung zu Fußnote 8). Doch was können Nichtwähler politisch beeinflussen? Nichts! Nur aktiv wählende Bürger haben es hingegen in der Hand, den potenzierten und weiter stattfindenden Machtmissbrauch elitärer Machtpolitiker Einhalt zu gebieten.

Die jüngste Vergangenheit und Gegenwart zeigt unmissverständlich, dass das harmonische Binom Autorität-Gehorsam zwischen Politiker und Bürger, was einst Früchte des Aufbaus und Wiederaufbaus unserer demokratischen Gesellschaft trug, zunehmend gestört ist. Das Potenzial des gestört Seins nimmt sowohl globale als auch lokale Züge an:

- Es ist die von einzelnen skrupellosen und geldgierigen Investment-Bankern ausgelöste Finanzkrise seit 2007, die Millionen Bürgern ihre Ersparnisse geraubt haben und von ihnen noch zusätzlich finanzielle Opfer fordert, für das sogenannte Stützen von „systemrelevanten“ Banken. ${ }^{4}$

n Es ist schließlich die kleinste gesellschaftliche Einheit, die Familie, der Zukunftsperspektiven geraubt werden. Es wird das Ersparte vernichtet, so dass Mittel für die familiäre

3 Beispiele für destruktive politische Aktivitäten - quer durch gesellschaftliche Bereiche - sind praxisnah nachvollziehbar bei Küppers (2013). Denken in Wirkungsnetzen, insb. Kap. 5, Tectum, Marburg.

4 Politisch bedeutet eine Bank sei systemrelevant, dass sie bei einer Störung (z. B. Insolvenz) mit ihr verbundene Banken in einen Strudel unkalkulierbaren Ausmaßes zieht, dessen gesellschaftliche, belastende Konsequenzen keiner überblickt. Evolutionär, auf der Basis realer komplexer Zusammenhänge, bedeutet systemrelevant, dass bei einer „Störung“ (z. B. Waldrodung, Wasserverschmutzung), das vernetzte System fähig ist, diese durch dynamisch stabile Rückkopplungseffekte auszugleichen. Die weitaus komplexeren Strukturen der Umwelt gegenüber den scheinbar komplexen, aber oft nur durch uns selbst erdachten, komplizierten Strukturen der Banken- und Finanzwelt lassen letztere in einem stark relativen Licht erscheinen. Die systemische Entwicklungsstrategie der Natur ist fundamental vernetzt. Sie stärkt nachhaltig und fehlertolerant das Überleben der Organismen. Rückgekoppelte Prozesse sind darin ein herausragendes, funktionales Merkmal. Die angeblich systemische Banken-Rettungsstrategie der Politik ist demgegenüber linear kausal. 
Sozialfürsorge zu Gunsten strauchelnder Banken wegfallen bzw. stark reduziert werden.

- Es ist die sich anschließende und ausweitende Weltwirtschaftskrise, die Millionen Bürgern Arbeitsplätze kostetet. Es ist nicht zuletzt die „Zukunft“ jeder Gesellschaft, die Jugend, vielfach ohne Aussicht, sich selbst zu verwirklichen in einer selbst gestalteten Gesellschaft.

- Politiker zeigen ihren Wählern im kommunalen lokalen Raum ihre imposanten und zugleich für die Bürger im Land desaströsen „Leistungsnachweise“ in Form von finanziell „aufgeblähten“ Großinvestitionen. Projekte wie der Flughafen Berlin-Brandenburg, der Bahnhofneubau Stuttgart21 oder die Hamburger Elbphilharmonie sind nur die Spitze des Eisberges (s. u.a. Bundesrechnungshof, Bemerkungen 2012, Landesrechnungshöfen, z. B. LRH-NRW 2012, Periodika des BdSt: Die öffentliche Verschwendung 2012, Däke, 2012).

- Der aktuelle Armuts- und Reichtumsbericht der Bundesregierung (2007-2011), mit durchschlagender, neoliberaler Handschrift ${ }^{5}$ ist - nach Aussagen von Bürger-Experten wie den Politikwissenschaftler Christoph Butterwegge - „... aufgehübscht..." (Weserkurier-Interview, S. 2, 7.3.2012). Der Historiker Hans-Ulrich Wehler spricht von „Soziale Ungleichheit stellt Legitimationsbasis der Bundesregierung infrage" (dradio-Interview vom 7.3.2013). Sie stellen den Politikern erneut ein katastrophales Zeugnis ihres Wählerauftrages „... Schaden vom Volk abzuwenden...“ aus.

Viele Bürger lassen sich nicht mehr von ihren Vertretern, den Politikern, „an der Nase herumführen“. Der Historiker Wehler geißelt in einem Interview (Spiegel-Online, 9.2.2013) „die wachsende Kluft zwischen Arm und Reich“. Präzisierend könnte man sagen, die wachsende Kluft zwischen versteckten und offenen Subventionen an Reiche und versteckten und offenen Kostenbelastungen an Arme. Wehler findet es „[...] überraschend, wie geduldig, ja phlegmatisch die Bundesbürger bislang damit umgehen". Den Streit um den aktuellen Armutsund Reichtumsbericht der Bundesregierung nennt Wehler dabei „ein schönes Beispiel für die Verlogenheit der Politik. Getäuscht wird am Ende der mündige Staatsbürger, dem man eine ehrliche Debatte offenbar nicht zumuten möchte". Zugleich fragt er sich: "Wie lange kann das gut gehen, diese extreme Verzerrung nach oben, ohne dass es politisch gefährlich wird?" Ihm wäre es "schon lieb, wenn der Ärger deutlicher artikuliert und debattiert würde [...]".

Überdeutlich steht die regierungspolitisch diktierte „Energiewende“ als ein Muster für organisatorische und kommunikative Destruktivität und Unfähigkeit sowie katastrophaler Planung und Lenkung; trotz grundlegend richtiger Entscheidung gegen Atomkraft und für regenerative Energien. Doch ein allgemeines Aufbegehren im Volk gegen die steigenden sozialisierten Kosten, wie z.B. die Erneuerbare-EnergieGesetz-Umlage (EEG-Umlage) im Energiesektor, bleibt aus.

Die Erkenntnis, dass eine Wirkung auch mehrere Ursachen besitzen kann, die Politiker nicht nach ihrem Gutdünken aus- blenden können, ist einer archetypische Erkenntnis in unserer Umwelt und Basis jeden nachhaltigen Fortschritts. Praxisbeispiele dafür, wie Hochachtsamkeit und (Selbst-Verantwortung) in unserem Gemeinwesen gesellschaftlichen Entscheidungs- und Handlungsraum realisiert werden kann, ja muss (!), werden in einem Folgebeitrag ausführlich erläutert.

\section{Das Land der Sehenden-Deutschland im Wahl-Herbst oder Deutschland im Herbst der Wahl?}

Aber folgen wir erst einmal wieder den Gedanken Saramagos: Deutschland schläft. Es ist die Nacht vor dem Wahltag. Die Bürger wählen am heutigen mechanisch-statistischen Herbstbeginn ihre Vertreter als Abgeordnete des Deutschen Bundestages. In Artikel 38 (1) des Grundgesetzes (GG) für die Bundesrepublik Deutschland (September 2010) heißt es u. a.:

„Sie (die Abgeordneten) sind Vertreter des ganzen Volkes, an Aufträge und Weisungen nicht gebunden und nur dem Gewissen unterworfen."

In unserem demokratischen Gemeinwesen scheint der Wahltag der einzige Tag einer Legislaturperiode zu sein, an dem die Politiker dem Souverän uneingeschränkt verfallen oder hörig sind. Denn „Zwischen den Wahlen [...] existiert Demokratie nur als potenzielle Bedrohung, dass die Volksvertreter oder ihre Partei nicht mehr wiedergewählt werden “ (Hobsbawn, 2009, S. 107).

Betrachten wir einmal klassisch die Wähler und Gewählten aus der Sicht eines Wirkungsnetzes, so haben wir es mit dem Muster einer Handlungsstruktur, eines Archetyps ${ }^{6}$ zu tun, der als "Tragödie der Allgemeingüter" (Senge, 2011, S. 461) bekannt ist. Abbildung 2 skizziert den Handlungsverlauf.

5 Die Partei der Freien Demokraten mit ihrem gegenwärtigen Vorsitzenden Rösler „manipulierte“ und verteidigte den Armuts- und ReichtumsBericht der Bundesregierung mit dem Titel „Lebenslagen in Deutschland“dadurch, dass verschiedene Passagen, wie z. B. „Privatvermögen in Deutschland sind sehr ungleich verteilt", im Abschlussbericht fehlen! Ebenso nicht mehr aufzufinden ist die Aussage, dass 2010 mehr als vier Millionen Menschen für einen Bruttostundenlohn von unter sieben Euro arbeiteten, sowie „Während die Lohnentwicklung im oberen Bereich positiv steigend war, sind die unteren Löhne in den vergangenen zehn Jahren preisbereinigt gesunken." (Lebenslagen in Deutschland, BMAS, 2013, Von der Leyen schließt die Schere, Süddeutsche.de, 6.6.2013).

6 Eine ganz wesentliche, machtvolle Erkenntnis, die aus der Analyse von komplexen Zusammenhängen - in der Natur ebenso wie in unserer von Menschen gestalteten Umwelt und Gesellschaft - gezogen wird, ist die der sich wiederholenden Mustererkennung, die Archetyp genannt wird. Senge (2011, S. 114) schreibt dazu: „Diese Systemarchetypen oder generische Strukturen sind der Schlüssel, wenn wir lernen wollen, die Strukturen in unserem persönlichen Leben und in Organisationen (wozu auch politische Organisationen zählen, d. A.) zu erkennen.". 


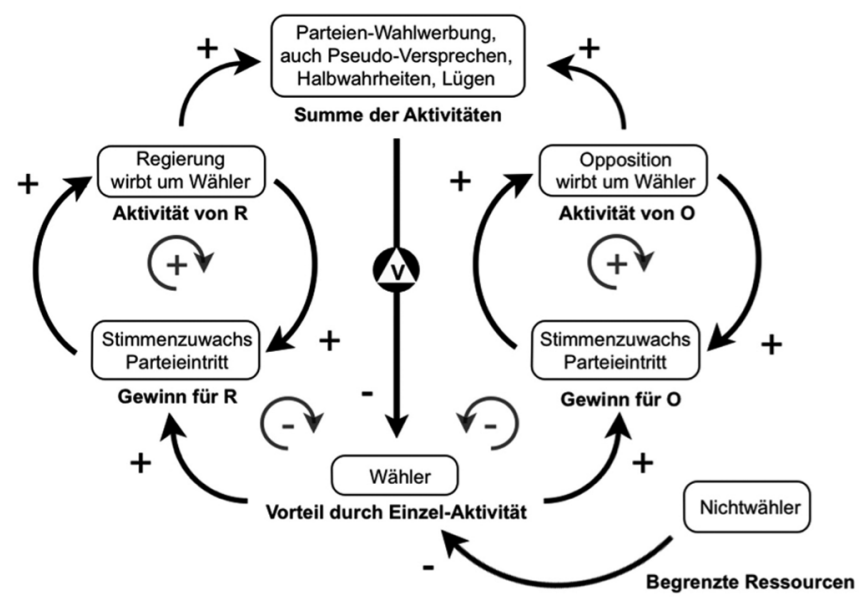

Abbildung 2: Tragödie der Allgemeingüter am Beispiel einer Wahl.

In periodischen Jahresabständen wählen die Bürger eines Landes ihre politischen Vertreter. Diese erhalten den Auftrag, das Land zum nachhaltigen Wohl der Bürger zu regieren - soweit die Theorie. Parteien werben auch außerhalb von Wahlzeiten mit ihren Wahlprogrammen, in denen sie mit - aus ihrer Sicht - vorteilhaften Vorschlägen zum Regieren die Wähler umgarnen. Sympathien der Wähler für regierende und opponierende Parteien sind vorhanden. Beide Gruppen schaffen sich auf diese Weise eine Stamm-Wählerschaft, die ausgebaut werden kann.

Die beiden Teufelskreise mit Wirkungsverstärkung (Dreiviertelkreise mit Plus-Zeichen) weisen darauf hin: Je mehr Aktivität Wähler zu werben, desto mehr Stimmenzuwachs und je mehr Stimmenzuwachs, desto mehr Wähler gewinnen die Gruppierungen. Der Wahlkampf zwischen beiden politischen Lagern um die Wählergunst wird umso schärfer ausgetragen, je näher der Wahltermin heranrückt. Die Summe der Aktivitäten, insbesondere auch die Qualitäten der Werbemethode mit ihren oft oberflächlichen und zweifelhaften Wahlversprechen führen zeitverzögert zu einem Wahlverdruss von Stammwählern und dem Verlust an Wählerstimmen auf beiden Seiten (in Abb. 2 durch den mittleren Pfeil mit inverser Wirkung von „Parteien-Aktivitäten“ zu „Wähler“ gekennzeichnet). Zusätzlich kann dieser Verlust noch verstärkt werden durch eine erhöhte Zahl von Nichtwählern. ${ }^{7}$

Dass die Parteien-,,Teufelskreise“ rechts und links mit der Verknüpfung der soeben beschriebenen inversen Wirkung in Abb. 2 Mitte, wieder - ganzheitlich gesehen - zu asymptotischen, relativ stabilen Verhältnissen von Wähler und Parteien führen, zeigen die beiden Dreiviertelkreise mit dem Minuszeichen. Vertrauen in die Politik, also in die Versprechen und Handlungen der Politiker, ist ein - wenn nicht sogar der Schlüssel für die Wähler, ihre Partei zu wählen.

Gut eine halbe Stunde vor Schließung der Wahllokale häufen sich die Spekulationen über das mögliche Wahlergebnis. Eine halbe Stunde nach deren Schließung zeigt sich bereits, was gemeinhin als unwahrscheinliches Ereignis gilt und von Marktforschungsinstituten jenseits jeder Statistik angesiedelt wird:
• Der Prozentsatz der Nichtwähler näherte sich der 45\%Marke!

- Noch schlimmer für die Parteien ist, dass insgesamt 30\% der wählenden Wahlberechtigten ihren Wahlzettel „weiß“, ohne jedes Kreuz und somit ungültig abgaben!

- Die beiden großen „Volksparteien“ (wann ist eine „Volkspartei“ groß und wann nicht mehr?) vereinigten dann nur noch $10 \%$ und $8 \%$ der Wahlberechtigten aus sich. Der Rest verteilte sich auf 5\%, $2 \%$ und $1 \%$ der anderen Parteien.

75\% aller Wahlberechtigten in Deutschland haben den Parteien und Politikern eine schallende Ohrfeige erteilt, für ihre Arbeit, die sie vorgeben im Auftrage des Volkes zu erbringen und mit der sie sich doch nur in ihrem ureigenen Interesse selbstverwirklichen wollen. Was ist zu tun? Wie sollen sich die beiden „Volksparteien“, die zu einem Häufchen Elend geschrumpft sind, verhalten?

„Das lassen wir uns nicht bieten“, hört man bereits trotzig Politiker aus den großen Verliererparteien populistisch durch die Medien rufen. Wir wollen umgehend Neuwahlen! Es heißt: Wer einmal die Staatsgewalt in Händen hat, wird sich vom Volk nicht Gesetze vorschreiben lassen. Für den britischen Politikwissenschaftler Colin Crouch zeigt sich darin ein merkwürdiges Paradox der politischen Klasse, der einerseits daran gelegen ist, die einfachen (nicht beunruhigten) Bürger davon abzuhalten, sich aktiv mit den Geheimnissen der Politik zu befassen. Zugleich ist sie aber eindringlich darum bemüht, dass eben jene Bürger sie passiv beim Akt der Stimmenabgabe unterstützen. Vor der wachsenden politischen Apathie suchen sie nach „Mitteln, diese Form der minimalen Beteiligung maximal auszureizen“ (Crouch, 2008, S. 143).

Unmittelbar nach der ersten Wahlhochrechnung breiten sich im ganzen Land spontane Massenveranstaltungen voller freudetrunkener Weißwähler aus. Sie können stolz von sich behaupten, ihren demokratischen Wahlauftrag erfüllt zu haben. Sie waren es, die den machtbesessenen Politikern, die sich nicht selten auf der untersten Stufe evolutionärer Erkenntnis $^{8}$ tummeln, mit durchgreifendem Erfolg die Leviten gelesen haben. Keine andere Wählergruppe! Die Macht ist wieder dahin zurückgekehrt, wo sie hingehört und von wo sie ausgeht, bei den Bürgern und Wählern des Landes.

Dreiviertel aller Wahlbürger verweigern sich denen zu folgen, die

- Vertrauen immer wieder durch Misstrauen ersetzen,

- sich wie in der Weltfinanzkrise keinen Deut ums demokratische Gemeinwesen kümmern,

7 vgl. u.a. http://de.wikipedia.org/wiki/Nichtwähler, darunter viele Einzelnachweise mit Grafiken über Tendenzen der Nichtwählergruppen bei Landes-, Bundes- und Europawahlen über mehrere Jahrzehnte

8 Hierzu schreibt der Neurobiologie G. Hüther (S. 113): „Erfolg macht blind, und die überstarke Kanalisierung des Denkens in einfachen Ursache-Wirkungs-Beziehungen hat ihren Preis. Menschen, die auf dieser Stufe der Erkenntnis stehenbleiben, halten alles, was sie als einfache Ursache-Wirkung-Beziehung erkannt haben, auch für machbar.". 
- Achtsamkeit aus ihrem Sprachschatz gestrichen haben bzw. durch Ignoranz verkleistern,

- und Verantwortung immer nur auf andere abwälzen, nur nicht bei sich selbst suchen.

Derartige Charakterzüge von Politikern sind einer Demokratie unwürdig. Das Handwerk eines Politikers ist kein Selbstzweck, aber viele politische Entscheidungsträger sehen es so. Saramago lässt in seinem Roman (S. 162) einen Bürgermeister sagen: „Einmal kommt die Stunde, in der wir entdecken, dass wir mehr wussten, als wir dachten “. Es war ein Bürgermeister, der keiner mehr sein wollte und daher dem politischen, am Boden liegenden System, spontan den Rücken zukehrte und der sich dem Demonstrationszug (vieler Weißwähler) in Richtung des Präsidentenpalastes anschloss.

Wären solche fiktiven Szenen politischen Bürgerwiderstandes bis zum schöpferisch-zerstörenden Machtgefüge unrealistisch? Unrealistisch in einem bürokratischen verkrusteten Apparat, in dem Angestellte und Beamte aufgrund ihres Dienstgrades nicht verpflichtet sind, „sich höheren Gedanken zu widmen oder unter der Oberfläche (der öffentlichen Verwaltung), nach verdächtigen Anzeichen zu suchen [...]“ (a.a.O. S. 242)? Wer würde sich bei dieser entropischen ${ }^{9}$ Entwicklung politischer Aktivitäten wundern, wenn im Herbst des Jahres, neben den nicht wenigen Bürgern, die bequem Wahlenthaltung üben, auch ein hoher Prozentsatz der Bürger WEISS wählen?

Bleibt Saramagos Parabel eine Fiktion in unserem demokratischen, sozialen Gemeinwesen? Sind Politiker zu lethargisch, sich den globalen, zielgerichteten Angriffen einer Finanz- und Wirtschaftsdiktatur zu entziehen? Haben Politiker, mitten im komplexen Krisengeschehen überhaupt den Willen oder die Chance, eine Politik zur Stärkung und fehlertoleranten Weiterentwicklung des Gemeinwesens durchzusetzen? Wenn dem so sein sollte, dann nur über den Weg einer wirkungsnetzorientierten Politik, über den Weg einer adaptiven Neuausrichtung ihres falsch programmierten Kausaldenkens und Handelns jenseits von Komplexitätsfallen und kurzfristigen Reparaturzyklen mit zunehmenden sozialen Folgen und Katastrophen.

\section{Literatur}

Bund der Steuerzahler (2012). Die öffentliche Verschwendung 2012. www.steuerzahler.de/Das-Schwarzbuch-zum-Download/ 15735c57166i475/index.html [abgerufen 22.7.2013].

Bundesrechnungshof (2012). Bemerkungen 2012. www.bundesrechnungshof.de/de/veroeffentlichungen/bemerkungen-jahresberichte/2012/ bemerkungen-2012-v 2 [abgerufen 22.7.2013].

Bundeswahlleiter (2008). Gültigkeit der Wahl. www.bundeswahlleiter.de/de/glossar/texte/Gueltigkeit_der_Wahl.html [abgerufen 22.7.2013].

Butterwegge, Christoph (2012). Interview mit dem Politikwissenschaftler. In Weserkurier 7. März., S. 2.

Crouch, C. (2008). Postdemokratie. Frankfurt a. M.: Edition Suhrkamp.
Däke, K. H. (2012). Die Milliarden Verschwender. München: Heyne.

Eurostat (2013). Unemployment Statistics. http:// epp.eurostat.ec.europa.eu/statistics_explained/index.php/Unemployment_statistics\#Youth_unemployment_trends [abgerufen 22.7.2013].

Hobsbawn, E. (2009). Globalisierung, Demokratisierung und Terrorismus. München: dtv.

Hüther, G. (2001). Bedienungsanleitung für ein menschliches Gehirn. Göttingen: Vandenhoeck \& Ruprecht.

Küppers, U. (2013). Denken in Wirkungsnetzen. Marburg: Tectum.

Landesrechnungshof Nordrhein-Westfalen (2012). Jahresbericht. www.lrh.nrw.de/LRHNRW_documents/Jahresbericht/

LRH_NRW_Jahresbericht_2012.pdf [abgerufen 22.7.2013].

N.N. (2013). Armutsbericht der Bundesregierung. Von der Leyen schließt die Schere. www.sueddeutsche.de/politik/armutsbericht-der-bundesregierung-von-der-leyen-schliesst-die-schere-1.1617044 [abgerufen 22.7.2013].

Saramago, J. (2009). Die Stadt der Sehenden. Reinbek: Rowohlt, 4. Aufl. Senge, P. (2011). Die Fünfte Disziplin. Stuttgart: Schäffer-Pöschel.

Stavrakis, G. (2013). Polizist spricht von aggressiver Stimmung. www.stuttgarter-nachrichten.de/inhalt.stuttgart-21-polizist-spricht-vonaggressiver-stimmung.ab4b6825-710e-4249-9bb5-09720c003d50.html [abgerufen 22.7.2013].

Teusch, Ulrich (2008). Die Katastrophengesellschaft. Warum wir aus Schaden nicht klug werden. Zürich: Rotpunktverlag.

Tuma, Thomas (2013). Extreme Verzerrung. Interview mit dem Historiker Hans-Ulrich Wehler In Der Spiegel, 7, 9. Februar. www.spiegel.de/ spiegel/print/d-90931283.html [abgerufen 22.7.2013].

Wehler, Hans-Ulrich (2013). Interview im dradio am 7. März.

Zeh, H. D. (2005). Entropie, S. 3, Fischer, Frankfurt a. M.

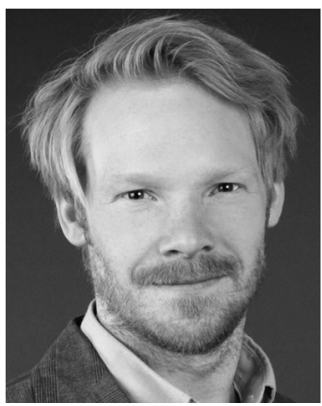

Jan-Philipp Küppers ist Sozialarbeiter, Politikwissenschaftler und Soziologe. Er studierte in Emden, Kiel und Zürich. Sein Interesse gilt Diskursen zu gesellschaftlichen Strukturfragen und eines gerechten Gemeinwesens. Zur Zeit lebt er in Vancouver, Kanada. Email: jan-philipp.kueppers@gmx.de.

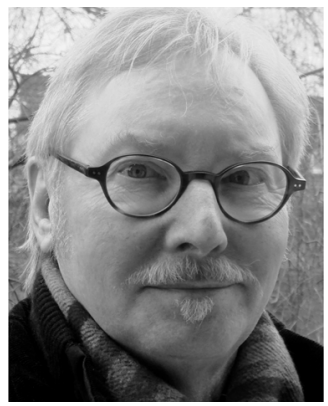

E. W. Udo Küppers ist promovierter Ingenieur, Bioniker und Systemiker. Er studierte Fertigungstechnik, Bionik und Evolutionsstrategie in Düsseldorf und Berlin. Unter KüppersSystemdenken - früher Küppers-Bionik - gilt sein Interesse Arbeiten im Grenzbereich zwischen Natur, Technik und Gesellschaft. Email: mail@udokueppers.de.

9 Entropie bezeichnet im ursprünglichen thermodynamischen Sinn ein „Maß für Unordnung“ (Zeh, 2005, S. 3). Hier soll es die Unordnung politischer Aktivitäten ausdrücken, die kurzsichtige, kausale Lösungen auf Kosten hoher sozialer Folgeprobleme - „Unordnung“ - eher favorisieren. 